\title{
A Retrospective Comparison of Haloperidol and Hydroxyzine Combination Therapy with Haloperidol Alone in the Treatment of Overactive Delirium
}

Junya Sato ( $\square$ junya02377@gmail.com )

International University of Health and Welfare: Kokusai Iryo Fukushi Daigaku https://orcid.org/00000002-2478-0190

Rei Tanaka

Shizuoka Cancer Center: Shizuoka Kenritsu Shizuoka Gan Center

\section{Research Article}

Keywords: delirium, haloperidol, hydroxyzine,Intensive Care Delirium Screening Checklist (ICDSC)

Posted Date: January 18th, 2022

DOI: https://doi.org/10.21203/rs.3.rs-1182527/v1

License: (c) (i) This work is licensed under a Creative Commons Attribution 4.0 International License.

Read Full License 


\section{Abstract \\ Background}

For the treatment of delirium, antipsychotics such as haloperidol are used as standard treatments. However, haloperidol has little sedative effect and may not be sufficiently effective in controlling overactive delirium. Hydroxyzine, an antihistamine, may be used in combination with haloperidol to supplement its sedative effect. Aim of this study was investigating the effect of haloperidol alone or in combination with hydroxyzine on the improvement of overactive delirium retrospectively.

\section{Method}

Delirium was assessed from medical records using the Intensive Care Delirium Screening Checklist (ICDSC). The number of patients and days with ICDSC score of $<4$, indicating absence of delirium after haloperidol alone or haloperidol and hydroxyzine were surveyed for 6 days.

\section{Results}

A total of 157 patients were diagnosed with delirium from April 2019 to July 2021, of which 18 patients received haloperidol alone, and 21 patients received the combination of haloperidol and hydroxyzine for overactive delirium. The number of patients with a mean ICDSC score of $<4$ on days $1-6$ were two patients $(11 \%)$ in the haloperidol groups and two patients $(10 \%)$ in the combination of haloperidol and hydroxyzine group $(P=0.999)$. The days within $<4$ of the ICDSC score on days $1-6$ were $0.8(1.3)$ and 0.8 (1.5), respectively $(P=0.848)$.

\section{Conclusion}

Haloperidol alone and haloperidol plus hydroxyzine are both effective in the treatment of overactive delirium. However, the concomitant use of hydroxyzine with haloperidol may not improve the efficacy of treatment of overactive delirium compared to haloperidol alone.

\section{Introduction}

Delirium is a problematic clinical symptom in terminally ill patients receiving palliative care. The etiology of delirium includes the presence of organic disorders such as old age, dementia, and cerebrovascular disease as possible preparatory factors. In addition to preparatory factors, physical symptoms such as pain, sleep disturbances, sensory deprivation, and physical restraints can promote the development of delirium. Finally, delirium is an acute cognitive disorder that can be triggered by dehydration due to infection as a precipitating factor, anemia, electrolyte abnormalities, and the use of inducing drugs such as opioids and benzodiazepines[1]. In overactive delirium, behavioral abnormalities can sometimes lead 
to dangerous behaviors such as falls, falling out of bed, or accidental tube removal. In addition, delirium becomes a daily occurrence, making home treatment difficult, prolonging hospitalization, and exhausting the medical staff who deal with delirious patients. The incidence of delirium itself is very low, around $1 \%$ in the general population[2], but it increases to $37 \%$ after surgery[3] and rises to $68 \%$ in terminal palliative care and to $88 \%$ in the $6 \mathrm{~h}$ before death[4]. Therefore, in providing medical care to patients at the end of life, delirium is a symptom that must be observed, and it must be prevented and treated.

Treatment of delirium is mainly through pharmacotherapy with antipsychotics. Haloperidol has been used as a standard drug for the treatment of delirium. However, typical antipsychotics are often followed by atypical antipsychotics due to concerns that typical antipsychotics increase the risk of death[5]. Atypical antipsychotics that are used include quetiapine, olanzapine, and risperidone[6-8]. Quetiapine and olanzapine have sedative effects due to histamine $\mathrm{H}_{1}$ receptor antagonism and are effective in controlling symptoms of delirium but are contraindicated in patients with diabetes or glucose intolerance. Risperidone is not limited by these glucose intolerance factors and is effective in improving hallucinations and delusional symptoms, but it is less sedating than quetiapine and olanzapine. The lack of injectable formulations of atypical antipsychotics is a barrier to their application in delirious patients who are unable to take them orally. Moreover, there is no clear evidence of superiority in efficacy against delirium[9]. Therefore, haloperidol is still a standard drug for the treatment of delirium. Haloperidol is administered orally, subcutaneous, or intravenously at a dose of 2-5 mg per dose, but it has a weak sedative effect on anger and excitement in hyperactive delirium. Therefore, haloperidol is sometimes combined with hydroxyzine, a histamine $\mathrm{H}_{1}$ receptor antagonist, to treat overactive delirium in clinical practice. However, the efficacy of haloperidol and hydroxyzine combination treatment compared to that of haloperidol alone has not been investigated. Thus, the aim of this study was to retrospectively compare the efficacy of haloperidol alone versus the combination of haloperidol and hydroxyzine for the treatment of delirium in hospitalized patients receiving palliative care.

\section{Methods}

\section{1) Subjects and interventions}

The study subjects were patients receiving palliative care who were admitted to the International University of Health and Welfare Hospital from April 2019 to July 2021. Of these patients, those who received antipsychotic medication for the treatment of hyperactive delirium were investigated. The treatments for delirium included in this study were haloperidol alone or haloperidol in combination with hydroxyzine. For patients with an intravenous route of administration, haloperidol (5-mg injection) or hydroxyzine (25-mg injection) was diluted in saline and administered intravenously over 15-30 min. If no intravenous route was available, these drugs were injected subcutaneously without dilution in saline. Oral administration was not performed. If the single dose was ineffective, one additional dose was allowed in a 3-h interval. The decision to select and add medications was made by the attending physician.

\section{2) Survey items and evaluation procedures}


The following items were investigated as part of the patient background. Gender, age, disease (cancer or non-cancer), performance status, preparatory factors (history of cerebrovascular disease, dementia, or heavy alcohol consumption), inducing factors (sleep rhythm disorder, presence of bed restraint, pain, respiratory distress, constipation, or urinary retention), and direct factors (opioid use, benzodiazepine use steroid use, benzodiazepine use, anticholinergic use, antihistamine use, dehydration, hypoxemia, infection/fever, hypercalcemia, renal dysfunction, abnormal liver function, brain metastasis, or hypoalbuminemia). Delirium was assessed through a review of medical records using the Intensive Care Delirium Screening Checklist (ICDSC)[10]. This checklist assessed the following eight symptoms every 24 h: (1) decreased level of consciousness, (2) lack of attention, (3) disorientation, (4) behavioral abnormalities associated with hallucinations and delusions, (5) risky behavior requiring physical restraint, (6) inappropriate conversation or emotion, (7) disturbance of sleep/wake cycle, and (8) diurnal variation in symptoms. Each item was scored as one point, and the full score was eight points. The cut-off value was four points or higher to be considered delirium. Patients with an ICDSC score of $\geq 4$ points before antipsychotic medication were eligible for evaluation. A score of four or less after antipsychotic administration was considered to be effective. The evaluation period was a maximum of $7 \mathrm{~d}$, including the pre-administration period. The primary efficacy endpoints were the number of patients and days of improvement in delirium. As a secondary endpoint, the changes in the ICDSC scores were compared.

\section{3) Statistical analysis}

The comparison of the continuous values between the haloperidol and the combination of haloperidol and hydroxyzine was made using an unpaired $t$-test. Fisher's exact test or Cochran-Armitage test was used for frequency comparisons. Multiple comparison tests (Dunnett test) were used to compare multiple periods before and after dosing. For each statistical method, a two-sided risk ratio of less than $5 \%$ was considered to be a statistically significant difference. The statistical analysis software used was BellCurve for Excel (Social Survey Research Information Co., Ltd. Tokyo).

\section{Results}

\section{1) Patient background}

There were 157 inpatients with a diagnosis of delirium during the study period. A total of 105 patients received any antipsychotics, excluding the 50 patients diagnosed with hypoactive delirium and the two patients diagnosed with delirium who improved without medication. Of these, 18 patients received haloperidol, and 22 patients received the combination of haloperidol and hydroxyzine. Among the patients who received the combination of haloperidol and hydroxyzine, one patient with a pre-dose ICDSC score of less than four points was excluded from the analysis. Analysis was performed on 18 patients in the haloperidol group and 21 patients in the combination of haloperidol and hydroxyzine group. The mean patient age (standard deviation, SD) in the haloperidol group and the haloperidol and hydroxyzine combination group was $82.8(7.9)$ and $72.7(11.1)$, respectively, with a significant difference $(P=0.003)$. There was also a tendency for the patient backgrounds of the two groups to differ in terms of gender and 
disease (cancer or non-cancer) $(P=0.062$ and $P=0.055)$. Therefore, a propensity score matching method was used to correct for the bias of these factors. There were nine patients in the haloperidol group and nine patients in the haloperidol and hydroxyzine combination group, respectively. The two groups were compared using the full analysis before propensity score matching as analysis 1 and the background correction analysis after propensity score matching as analysis 2 . The characteristics of the patients are shown in Table 1.

\section{2) Patients number within $<4$ of ICDSC score}

In analysis 1 , the number of patients with a mean ICDSC score of $<4$, indicating improvement in delirium, on days $1-6$ were two patients $(11 \%)$ in the haloperidol group and two patients $(10 \%)$ in the haloperidol and hydroxyzine combination group, showing no significant difference between the two groups $(P=$ 0.999 , Table 2). Also, in analysis 2 , the number of patients with a mean ICDSC score of $<4$ was one patient $(11 \%)$ in the haloperidol group and zero patients $(0 \%)$ in the haloperidol and hydroxyzine combination group, with no difference between the two groups $(P=1.000)$.

\section{3) Days within $<4$ of the ICDSC score}

In analysis 1 , the days within $<4$ of the ICDSC score on days $1-6$ were 0.8 (1.3) in the haloperidol group and 0.8 (1.5) in the haloperidol and hydroxyzine combination group, showing no significant difference between the two groups $(P=0.848$, Table 3$)$. These results for the two groups were also observed to have no difference in analysis 2 . Also, in analysis 2 , these days were $0.7(0.8)$ and $0.3(0.7)$ for the haloperidol and haloperidol and hydroxyzine combination groups, respectively, with no difference between the two groups $(P=0.241)$.

\section{4) Changes in the IDCSC scores}

In analysis 1, the mean IDCSC scores (SD) on the pre-treatment in the haloperidol group and the haloperidol and hydroxyzine combination group were $7.2(0.9)$ and $6.8(1.1)$, respectively, with no significant difference between the two groups $(P=0.218)$. The IDCSC scores in the haloperidol group were $6.0(1.7)$ and $5.5(2.0)$ on days $1-3$ and $4-6$, respectively, showing a significant decrease compared to that of the pre-treatment $(P=0.038$ and $P=0.009)$. The mean score on days $1-6$ was $6.0(1.6)$, showing a significant decrease compared to that of the pre-treatment $(P=0.010)$. The IDCSC scores in the haloperidol and hydroxyzine combination group were $5.8(1.5)$ and $5.7(1.8)$ on days $1-3$ and $4-6$, respectively, showing a trend toward lower scores compared to that of the pre-treatment $(P=0.077$ and $P$ $=0.050$ ). The mean IDCSC score on days $1-6$ was $5.8(1.4)$, showing a significant decrease compared to that of the pre-treatment $(P=0.019)$. There was no significant difference in the IDCSC scores between the two groups on days $1-3,4-6$, and $1-6$, respectively $(P=0.794, P=0.839$, and $P=0.806)$ (Figure 1 ).

In analysis 2, the mean IDCSC scores (SD) on the pre-treatment in the haloperidol group and haloperidol and hydroxyzine combination group were $7.1(0.8)$ and $6.9(1.1)$, respectively, with no significant difference between the two groups $(P=0.618)$. The mean IDCSC score in the haloperidol group was 5.8 (1.6) and $5.0(2.8)$ on days $1-3$ and $4-6$, respectively $(P=0.263$ and $P=0.076)$. The IDCSC score was 
$5.7(1.8)$ on days $1-6(P=0.092)$, and there was a tendency for the IDCSC score to decrease on days 4- 6 and days $1-6$. The mean IDCSC scores in the haloperidol and hydroxyzine combination group were 6.3 (1.4), $6.5(0.6)$, and $6.6(1.0)$ on days $1-3$, days $4-6$, and days $1-6$, respectively, and none of which showed a significant decrease in the IDCSC score compared to that of the pre-treatment $(P=0.515, P=$ $0.702, P=0.536)$. There was no significant difference in the IDCSC scores between the two groups on days $1-3,4-6$, and $1-6$, respectively $(P=0.543, p P=0.319$, and $P=0.268)$ (Figure 2).

\section{5) Adverse events}

No extrapyramidal symptoms such as tremor, parkinsonian symptoms, muscle rigidity, akathisia, or neuropsychiatric symptoms such as agitation or excessive sedation were observed in the haloperidol and/or hydroxyzine administered patients.

\section{Discussion}

Haloperidol has been used as a key drug in the treatment of delirium. However, haloperidol has little sedative effect and may lack efficacy in the treatment of delirium with agitation. In a randomized controlled trial (RCT) of haloperidol ( $2 \mathrm{mg}$ ) alone and in combination with lorazepam ( $3 \mathrm{mg}$ ), haloperidol alone was associated with a decrease in the Richmond Agitation-Sedation Scale (RASS) score of -2.3 points, and the final RASS score ranged from 0 to 1 (clear consciousness to somnolence at the level of awakening to a call)[11]. In contrast, the lorazepam combination was associated with a -4.1 point reduction in RASS score, and the final RASS score ranged from -2 to -3 (moderate sedation, responsive to calls). If the effect of haloperidol is poor, additional doses of haloperidol or concomitant use of benzodiazepines may be used. However, overdose of haloperidol is associated with concerns of aspiration, drug-induced parkinsonism, and cardiotoxicity. In addition, concomitant use of benzodiazepines may cause respiratory depression. For example, flunitrazepam-induced respiratory depression in palliative care patients has been reported to be observed in $17 \%$ of patients[12]. In particular, delirious patients with cancer pain are more likely to use opioid analgesics. Concomitant use of opioids and benzodiazepines increases the risk of excessive respiratory depression[13]. These are the dilemmas of treating delirium with haloperidol and benzodiazepine. With these backgrounds, hydroxyzine is sometimes used concomitantly to complement the sedative effect of haloperidol.

Hydroxyzine is classified as a first-generation antihistamine and permeates the blood-brain barrier. It inhibits the action of histamine in the thalamus, hypothalamus, and limbic system of the central nervous system and has anxiolytic and sedative effects. The maximum blood concentration of hydroxyzine is reached in about $2 \mathrm{~h}$ after intramuscular injection, and it is long-acting, with a duration of action of 6 to $24 \mathrm{~h}$. Hydroxyzine is used clinically for sedation and insomnia in patients who have difficulty using haloperidol due to Parkinson's disease or benzodiazepines due to respiratory instability. However, the efficacy of the combination of haloperidol and hydroxyzine in the treatment of delirium has not been reported previously. 
The present study showed that both haloperidol alone and hydroxyzine combination treatment were effective in reducing the IDCSC score and improving delirium. However, no evidence was presented that the concomitant use of hydroxyzine beneficially contributes to the efficacy of haloperidol. Despite the negative results, this is the first paper to evaluate the efficacy of the combination of haloperidol and hydroxyzine for the treatment of delirium compared to that of haloperidol alone. There are several possible reasons for the lack of additional effects of hydroxyzine in this study. Most of the subjects in this study were elderly patients with poor general conditions (performance status $\geq 3$ ), with an average age of around 80 years. Many patients had delirium preparatory factors such as dementia or cerebrovascular disease. Both treatment groups were patients with severe delirium, with a pre-treatment ICDSC score of around seven, which may have resulted in a lack of therapeutic effect of these drugs. The elimination half-life of haloperidol and hydroxyzine are long, over $20 \mathrm{~h}$ and $14 \mathrm{~h}$, respectively. However, in a study comparing the effects of haloperidol and chlorpromazine in patients with refractory delirium, haloperidol was administered every $4 \mathrm{~h}[14]$. The pattern of antipsychotic use in the present study was mostly after the onset of nocturnal agitation or prophylactic administration once before falling asleep, or addition during early morning relapse after administration the night before. There was no multiple scheduled daily administration of antipsychotic medication. Our use of haloperidol in the treatment of delirium may have needed to be revisited. Antihistamines are reported to be risk agents for delirium[15]. Although the suspect drug is diphenhydramine, case-control studies and cohort studies have reported the delirium-inducing effects of antihistamines[16, 17]. Thus, in situations where the effect of haloperidol is insufficient, the sedative effect of concomitant hydroxyzine may not be sufficient. In such cases, there is little benefit to adding hydroxyzine for delirium.

The limitations of this study are as follows. First, this study is a retrospective observation of a small number of patients at a single institution. During the study period, 37\% (39 patients) of the patients with delirium received haloperidol alone or in combination with hydroxyzine, but the patient backgrounds of the two groups differed in terms of age, disease, and gender. These were adjusted using the propensity score matching method, resulting in an analysis of 18 patients, which may have reduced the power of detection. Second, the choice of haloperidol alone or in combination with haloperidol and hydroxyzine was determined by the patient's physician. Many of the physicians selected did not specialize in the field of psychiatry, and there is uncertainty about the reasons for their drug selection. In the future, prospective comparative studies that improve the weaknesses of this study are needed to evaluate the effect of haloperidol plus hydroxyzine combination on the treatment of delirium.

In summary, hydroxyzine may be used in combination with haloperidol to add sedative effects in the treatment of overactive delirium. However, it is possible that there is little benefit to using hydroxyzine in combination with haloperidol. Further detailed investigations on concomitant medications when the effect of haloperidol on delirium is insufficient are needed.

\section{Declarations}

\section{Acknowledgements}


We thank Yu Yamada, MD, Ph.D., Mayumi Tsukagoshi, CNS, Daiki Hasegawa, Momoka Suda, and Ayumi Ozawa for their help with data collection and expert advice on this study.

\section{Funding}

The authors declare that no funds, grants, or other support were received during the preparation of this manuscript.

\section{Competing Interests}

The authors have no relevant financial or non-financial interests to disclose.

\section{Availability of data and material}

All data generated or analysed during this study are included in this published article.

\section{Code availability}

Not applicable.

\section{Author Contributions}

Junya Sato and Rei Tanaka contributed to the conception and design of the study. Junya Sato conducted the data collection and initial analysis. Rei Tanaka participated in additional analysis of the data and logical discussion of the results. The first draft of the manuscript was written by Junya Sato and read by Rei Tanaka, which was revised and agreed upon by both authors.

\section{Ethics approval}

This study was conducted in accordance with the principles of the Declaration of Helsinki. It was approved by the Ethics Committee of International University of Health and Welfare (Date: April 21, 2021/No. 21-B-457).

\section{Consent to participate}

Not applicable.

\section{Consent to publish}

Not applicable.

\section{References}

1. American Psychiatric Association, DSM-5 Task Force (2013) Diagnostic and statistical manual of mental disorders: DSM-5 ${ }^{\mathrm{Tm}}$, 5th edn. American Psychiatric Publishing, Inc., Washington 
2. Folstein MF, Bassett SS, Romanoski AJ, Nestadt G (1991) The epidemiology of delirium in the community: the Eastern Baltimore Mental Health Survey. Int Psychogeriatr 3:169-176

3. Dyer CB, Ashton CM, Teasdale TA, Postoperative (1995) delirium A review of 80 primary datacollection studies. Arch Intern Med 155: 461-5

4. Lawlor PG, Gagnon B, Mancini IL, Pereira JL, Hanson J, Suarez-Almazor ME, Bruera ED (2000) Occurrence, causes, and outcome of delirium in patients with advanced cancer: a prospective study. Arch Intern Med 160:786-794

5. Huybrechts KF, Gerhard T, Crystal S, Olfson M, Avorn J, Levin R, Lucas JA, Schneeweiss S (2012) Differential risk of death in older residents in nursing homes prescribed specific antipsychotic drugs: population based cohort study. BMJ 344:e977

6. Tahir TA, Eeles E, Karapareddy V, Muthuvelu P, Chapple S, Phillips B, Adyemo T, Farewell D, Bisson JI (2010) A randomized controlled trial of quetiapine versus placebo in the treatment of delirium. J Psychosom Res 69:485-490

7. Breitbart W, Tremblay A, Gibson C (2002) An open trial of olanzapine for the treatment of delirium in hospitalized cancer patients. Psychosomatics 43:175-182

8. Kishi Y, Kato M, Okuyama T, Thurber S (2012) Treatment of delirium with risperidone in cancer patients. Psychiatry Clin Neurosci 66:411-417

9. Burry L, Hutton B, Williamson DR, Mehta S, Adhikari NK, Cheng W, Ely EW, Egerod I, Fergusson DA, Rose L (2019) Pharmacological interventions for the treatment of delirium in critically ill adults. Cochrane Database Syst Rev 9:CD011749

10. Bergeron N, Dubois MJ, Dumont M, Dial S, Skrobik Y (2001) Intensive Care Delirium Screening Checklist: evaluation of a new screening tool. Intensive Care Med 27:859-864

11. Hui D, Frisbee-Hume S, Wilson A, Dibaj SS, Nguyen T, De La Cruz M, Walker P, Zhukovsky DS, Delgado-Guay M, Vidal M, Epner D, Reddy A, Tanco K, Williams J, Hall S, Liu D, Hess K, Amin S, Breitbart W, Bruera E (2017) Effect of lorazepam with haloperidol vs haloperidol alone on agitated delirium in patients with advanced cancer receiving palliative care: A randomized clinical trial. JAMA 318:1047-1056

12. Matsuo N, Morita T (2007) Efficacy, safety, and cost effectiveness of intravenous midazolam and flunitrazepam for primary insomnia in terminally ill patients with cancer: a retrospective multicenter audit study. J Palliat Med 10:1054-1062

13. Baillargeon J, Singh G, Kuo YF, Raji MA, Westra J, Sharma G (2019) Association of opioid and benzodiazepine use with adverse respiratory events in older adults with chronic obstructive pulmonary disease. Ann Am Thorac Soc 16:1245-1251

14. Hui D, De La Rosa A, Wilson A, Nguyen T, Wu J, Delgado-Guay M, Azhar A, Arthur J, Epner D, Haider A, De La Cruz M, Heung Y, Tanco K, Dalal S, Reddy A, Williams J, Amin S, Armstrong TS, Breitbart W, Bruera $E$ (2020) Neuroleptic strategies for terminal agitation in patients with cancer and delirium at an acute palliative care unit: a single-centre, double-blind, parallel-group, randomised trial. Lancet Oncol 21:989-998 
15. Tuma R, DeAngelis LM (2000) Altered mental status in patients with cancer. Arch Neurol 57:17271731

16. Marcantonio ER, Juarez G, Goldman L, Mangione CM, Ludwig LE, Lind L, Katz N, Cook EF, Orav EJ, Lee TH (1994) The relationship of postoperative delirium with psychoactive medications. JAMA 272:1518-1522

17. Agostini JV, Leo-Summers LS, Inouye SK (2001) Cognitive and other adverse effects of diphenhydramine use in hospitalized older patients. Arch Intern Med 161:2091-2097

\section{Tables}

Due to technical limitations, table 1,2,3 is only available as a download in the Supplemental Files section.

\section{Figures}

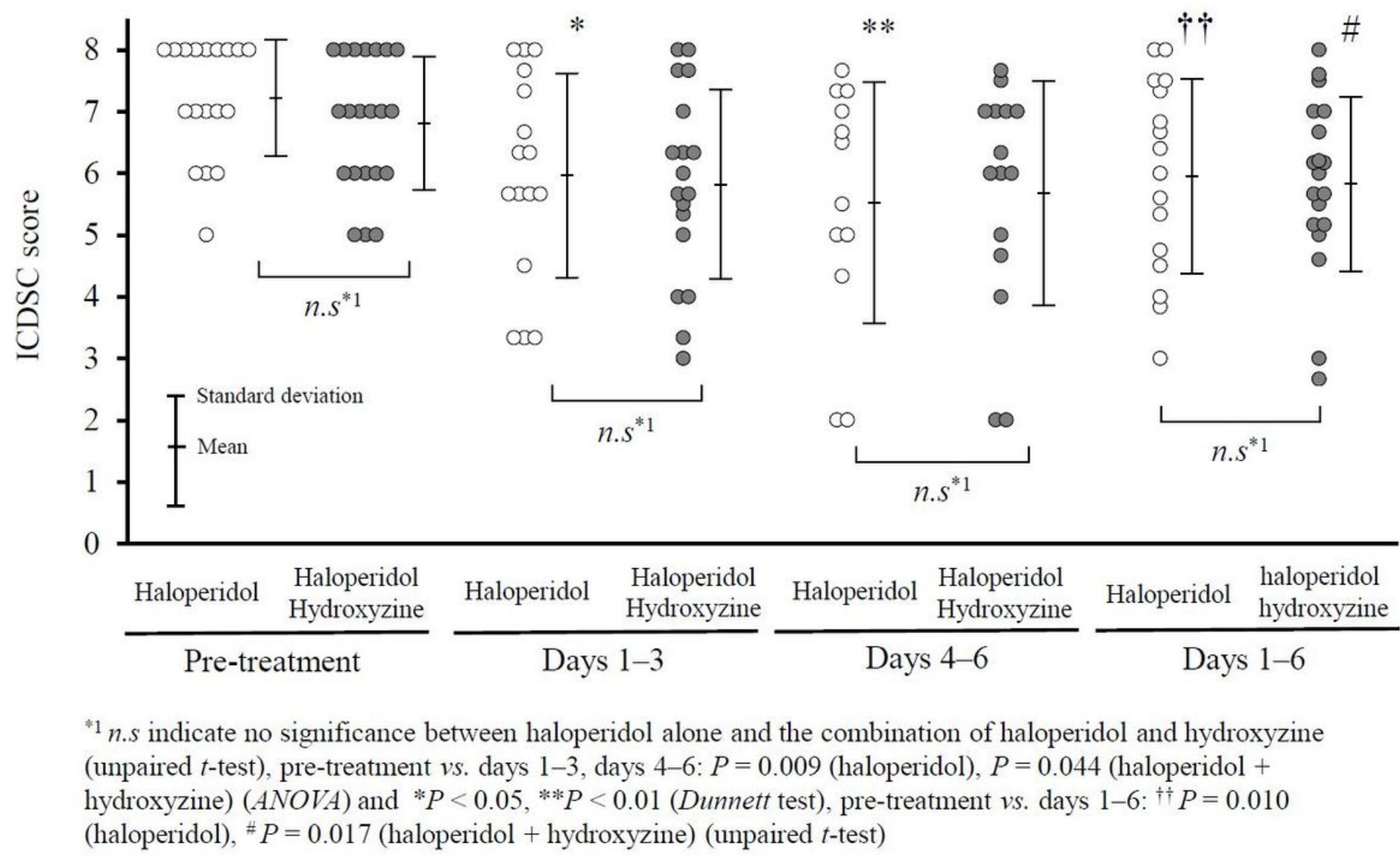

\section{Figure 1}

Changes in the Intensive Care Delirium Screening Checklist (ICDSC) scores before and after treatment with haloperidol alone and combination of haloperidol and hydroxyzine in analysis 1 . 


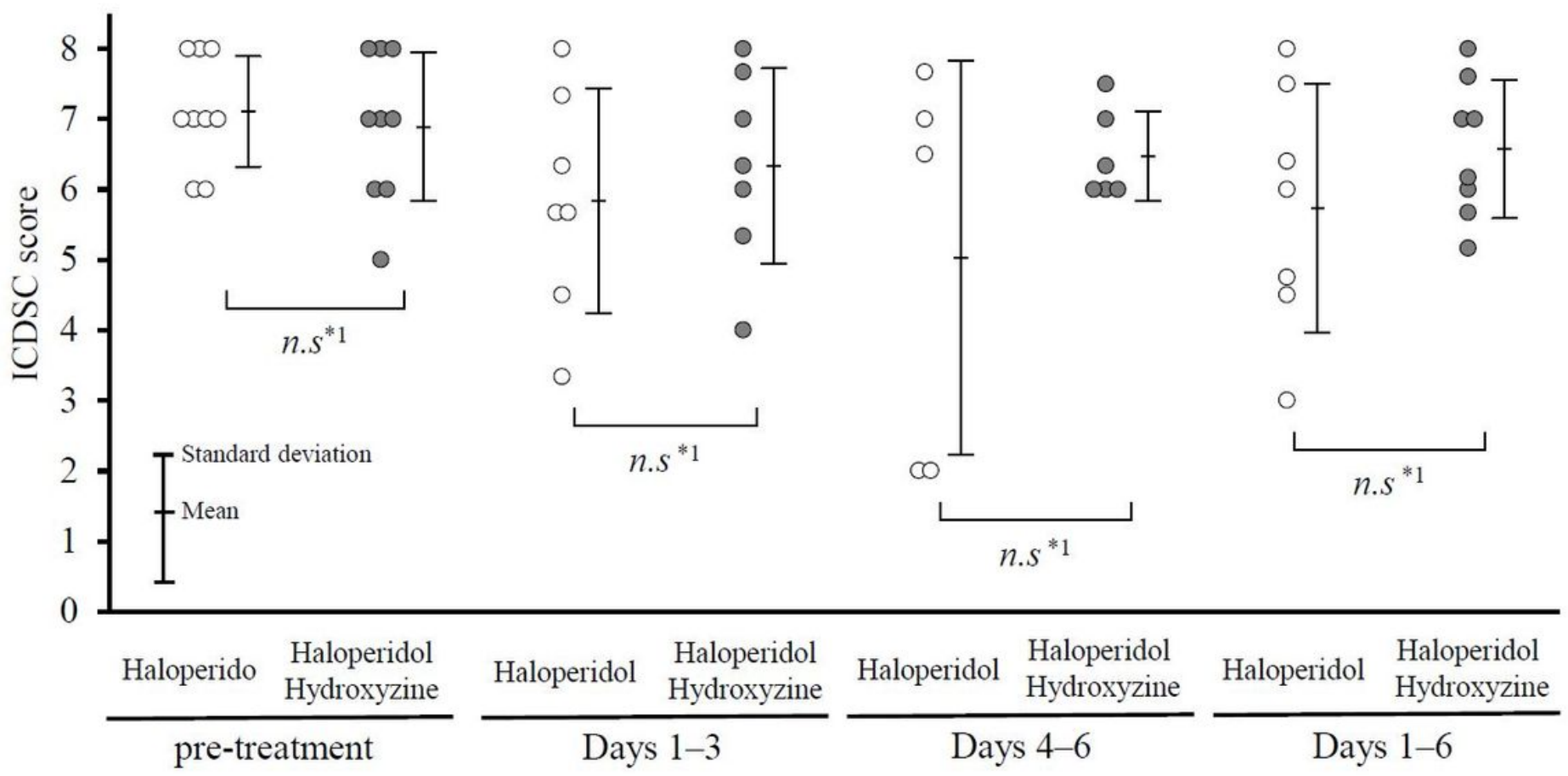

${ }^{*}$ n.s indicate no significance between haloperidol alone and combination of haloperidol and hydroxyzine (unpaired $t$-test), pre-treatment vs. days 1-3, days 4-6: $P=0.097$ (haloperidol), $P=0.575$ (haloperidol + hydroxyzine) (ANOVA), pre-treatment vs. days 1-6: $P=0.126$ (haloperidol), $P=0.537$ (haloperidol + hydroxyzine) (unpaired $t$-test)

Figure 2

Changes in the Intensive Care Delirium Screening Checklist (ICDSC) scores before and after treatment with haloperidol alone and combination of haloperidol and hydroxyzine in analysis 2 .

\section{Supplementary Files}

This is a list of supplementary files associated with this preprint. Click to download.

- 2021.12.18Table.1.pdf

- 2021.12.18Table.1continued.pdf

- 2021.12.18Table.2.pdf

- 2021.12.18Table.3.pdf 